\title{
La respuesta estatal a la violencia intrafamiliar
}

\author{
Lidia Casas Becerra* \\ Macarena Vargas Pavez**
}

\begin{abstract}
RESUMEN
En Chile el tratamiento y respuesta al fenómeno de la violencia intrafamiliar ha cambiado sustancialmente desde la primera ley dictada en 1994 hasta la fecha. Se ha transitado desde un enfoque terapéutico hacia uno de respuestas más represivas, entregando con ello competencia para conocer de estos actos al sistema de justicia penal. Indagar, desde una perspectiva cuantitativa y cualitativa, acerca del impacto de las modificaciones legales de los últimos años es el objeto del presente trabajo.
\end{abstract}

Violencia intrafamiliar - administración de justicia respuesta estatal

\section{The state response to domestic violence}

\begin{abstract}
In Chile, treatment and response to the phenomenon of the domestic violence has substantially changed since the first statutory law enacted in 1994 to nowadays. It has gone from a therapeutic approach towards a more repressive response, thereby giving jurisdiction to the criminal justice over this kind of act. To inquire, from a quantitative and qualitative approach about the impact of the legal changes of the last years is the object of this work.
\end{abstract}

Domestic violence - justice application - state response

* Abogada, profesora e investigadora de la Facultad de Derecho de la Universidad Diego Portales, Santiago de Chile. lidia.casas@udp.cl

** Abogada, Magíster en Gestión y Políticas Públicas, profesora e investigadora de la Facultad de Derecho de la Universidad Diego Portales, Santiago de Chile. macarena.vargas@udp.cl

Artículo recibido el 30 de marzo de 2011 y aceptado para su publicación por el Comité Editorial el 27 de mayo de 2011. 


\section{INTRODUCCIÓN}

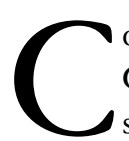

on la dictación de la Ley $\mathrm{N}^{\circ} 19.325$ en 1994 se reconoce por primera vez en Chile que la violencia intrafamiliar es una conducta merecedora de reproche social ${ }^{1}$. Este reconocimiento obedeció a un contexto sociopolítico de adopción de medidas legislativas y de políticas públicas en la región destinadas a combatir la violencia en contra de las mujeres, considerando este tipo de conductas como una grave violación a los derechos humanos ${ }^{2}$.

Desde la promulgación de dicha ley a la fecha el número de casos conocidos por los tribunales de justicia fue en paulatino aumento. La Encuesta Nacional de Victimización por Violencia Intrafamiliar y Delitos Sexuales del 2008 arrojó que un 35,7\% de las mujeres entre 18 y 65 años que han tenido una relación de convivencia fue víctima de algún tipo de violencia por parte de sus parejas, cuyas expresiones preponderantes son la combinación de violencia física, sicológica y sexual $(32,6 \%)$ y la sicológica y física $(30,5 \%)^{3}$. En los últimos años, sin embargo, la violencia contra la mujer ha sido visibilizada por sus manifestaciones más graves a través de lo que se conoce como femicidio, el asesinato de mujeres de manos de sus parejas ${ }^{4-5}$.

Estas cifras, no obstante, no evidencian necesariamente un aumento de la prevalencia de violencia en contra de la mujer, sino la reducción de la cifra negra, en otras palabras, un aumento del número de casos denunciados. En efecto, estudios realizados hace más de 20 años muestran que denunciar a la pareja por actos violentos no parecía ser una conducta avalada socialmente. Una investigación realizada en 1989 mostró que un $84 \%$ de las mujeres violentadas no denunciaba, entre otras, por razones de "privacidad" o "porque no se saca nada" 6 . Las mujeres debieron sortear grandes obstáculos culturales para reclamar de las agresiones de las cuales fueran objeto, dado que la idea de que "la ropa sucia se lava en casa” era (y probablemente lo siga siendo) parte del imaginario colectivo $^{7-8}$.

${ }^{1}$ Ley $\mathrm{N}^{\circ} 19.325$ publicada en el Diario Oficial 4 de agosto de 1994.

${ }^{2}$ OEA, Acceso a la justicia para las mujeres víctimas de violencia en las Américas, Comisión Interamericana de Derechos Humanos, Washington, DC, 2007. OEA/Ser.L/V/II.Doc.68 20 enero de 2007.

${ }^{3}$ Ministerio del Interior, Encuesta Nacional de Victimización por Violencia Intrafamiliar y Delitos Sexuales 2008. En: http://www.seguridadpublica.gov.cl/files/presentacion_violencia_intrafamiliar_v2.pdf [citado en 23 de septiembre 2010].

${ }^{4}$ SERNAM, Femicidios 2008. En: <http://www.sernam.cl/portal/index.php/femicidios-2008>. Visitado el 20 de noviembre de 2009.

${ }^{5}$ Rodríguez, R., “Análisis estadístico descriptivo de los femicidios ocurridos en el año 2007”, Revista Jurídica del Ministerio Público $\mathrm{N}^{\circ} 35$, julio 2008, p. 336.

${ }^{6}$ Moltedo, C., Silva, C., Orellana, C. et al., Estudio sobre violencia doméstica en mujeres pobladoras chilenas, Santiago, 1989, p. 14.

${ }^{7}$ Casas, L. y Mera, A., Violencia de género y Reforma Procesal Penal chilena. Delitos sexuales y lesiones, Cuaderno de Análisis Jurídico, Serie de Publicaciones Especiales 16, Facultad de Derecho Universidad Diego Portales y el Centro de Estudios de Justicia para las Américas, Santiago, 2004.

${ }^{8}$ Provoste, P., Violencia contra la mujer en la pareja: respuestas de la salud pública en Santiago de Chile, Serie Mujer y Desarrollo 85, CEPAL, Santiago, 2007, p. 13. 
Con todo, la primera ley de violencia abrió el reconocimiento del problema, lo cual claramente fue un avance: entregaba herramientas para dictar medidas cautelares, propiciaba medidas alternativas a la pena-sanción, como el trabajo comunitario, e instalaba la idea de una intervención psicosocial para los agresores. Esta ley tuvo un claro enfoque terapéutico bajo la premisa de que era necesario buscar la reconciliación y la reparación de los vínculos familiares afectados por la violencia a través de la intervención judicial, específicamente mediante la conciliación ${ }^{9-10}$. Estudios de la época sobre la aplicación de la Ley $\mathrm{N}^{\circ} 19.325$ mostraron que la mayoría de los casos (92\%) terminaba por un avenimiento instado por el tribunal ${ }^{11}$, lo que a juicio de los especialistas implicaba la ausencia de sanciones a los ofensores, perpetuaba los estereotipos de género y justificaba la violencia ${ }^{12}$.

En el año 2005 con la dictación de la Ley $N^{\circ} 19.968$ que crea los tribunales de familia se establece un nuevo procedimiento para los casos de violencia intrafamiliar y se elimina el llamado a conciliación ${ }^{13}$. Sin embargo, se establece la figura de la suspensión condicional de la dictación de la sentencia como forma de poner término al proceso. Esta figura supone algún nivel de acuerdo entre las partes e, incluso en algunos casos, la derivación a una instancia de mediación ${ }^{14}$.

Este enfoque terapéutico cambia radicalmente con la Ley No 20.066 de 2005 que introduce el delito de maltrato habitual, dando paso así a la adopción de respuestas más represivas ${ }^{15}$. Si bien el sistema penal conocía de actos de violencia intrafamiliar, la especificidad de género que éstos presentaban no quedaba en evidencia al ingresar bajo lesiones, amenazas, violación de morada, delitos de daños u homicidios ${ }^{16}$.

${ }^{9}$ Casas, L., "Ley N 20.066 sobre Violencia Intrafamiliar: ¿un cambio de paradigma?”, en Anuario de Derechos Humanos 2006, Universidad de Chile, p. 198.

${ }^{10}$ Ello no es nuevo en el derecho comparado y, como advierte Siegel, los primeros esfuerzos en los Estados Unidos por sancionar la violencia doméstica jueces y asistentes sociales favorecieron, por vías formales e informales, la preservación de la unificación familiar. Siegel, R., "Regulando la Violencia marital", trad. Roberto Gargarella, en Derechos y Grupos desaventajados, Gargarella, R., comp., Yale Law School, Universidad de Palermo, y GEDISA Editorial, Barcelona, 1999, pp. 83-84.

${ }^{11}$ Centro de Ética, Universidad Alberto Hurtado, Informe Ethos Violencia Intrafamiliar No 22. 2000.

${ }^{12}$ Estudios desarrollados principalmente por el Instituto de la Mujer, Estudio de seguimiento de la Ley de Violencia Intrafamiliar, Informe Final, Santiago, agosto de 1995, mimeo; Rioseco, L., Evaluación de la Ley de Violencia Intrafamiliar 19.325 y una propuesta para su modificación, Fundación Instituto de la Mujer, Santiago, 1997, mimeo; Casas, L., Dides, C. y Magaña, A., Estudio sobre la sistematización de información sobre el control de medidas y sanciones impuestas en virtud del art. 5 de la Ley $\mathrm{N}^{\circ} 19.325$, Informe Final para el Sernam, Santiago, 2001, mimeo.

${ }^{13}$ Ley $\mathrm{N}^{\circ} 19.968$ publicada en el Diario Oficial 30 de agosto de 2004.

${ }^{14} \mathrm{La}$ ley dispone que los casos de violencia podrán ser sometidos a mediación sólo si hay reconocimiento de los hechos fundantes de la demanda o denuncia, si no existen antecedentes previos de violencia en contra del demandado o denunciado independiente de la víctima o condena por algún crimen o simple delito contra las personas, delitos sexuales o cuando el juez estime conveniente con la continuación del proceso, todo ello conforme a iguales requisitos respecto de la suspensión de la dictación de la sentencia.

${ }^{15}$ Ley $\mathrm{N}^{\circ} 20.066$ publicada en el Diario Oficial el 7 de octubre de 2005.

${ }^{16}$ Casas y Mera, op. cit. A lo más, eran caracterizados y tratados como "delitos pasionales" sin que se entendiera a cabalidad el fenómeno de violencia subyacente. 
Con esta nueva normativa la respuesta estatal se amplifica dando paso a la intervención de jueces de familia, de fiscales y jueces de garantía, cuando el caso posea -a juicio de los primeros- los caracteres de frecuencia e intensidad que configuren la habitualidad de los actos violentos. Si ello es así, los jueces de familia se declararán incompetentes y derivarán los antecedentes a la fiscalía que corresponda dando paso al conocimiento y solución de este tipo de conflictos por la vía penal.

En este contexto, el presente trabajo tiene como objetivo indagar acerca del tratamiento y respuesta que reciben los casos de violencia doméstica en el sistema de administración de justicia chileno. En otras palabras, busca responder las interrogantes que siguen: ¿Cómo terminan las causas de violencia intrafamiliar en sede penal y familiar? ¿Se advierten diferencias en materia de medidas cautelares y de las condiciones de las salidas alternativas? ¿Cuál es el impacto de transitar desde un enfoque terapéutico a uno más represivo?

En las páginas que siguen se presenta parte de los resultados de una investigación empírica realizada durante el año 2009 que analiza el fenómeno de la violencia de género en la administración de justicia, tanto desde una perspectiva cuantitativa como cualitativa. Este estudio contempló, además del levantamiento y examen de estadísticas judiciales, la revisión de 140 carpetas de tribunales de familia de las Regiones V y Metropolitana y 148 carpetas del Ministerio Público de tres fiscalías de la Región Metropolitana (Occidente, Oriente y Centro Norte) y dos de la V Región (Valparaíso y Viña del Mar $)^{17}$.

Para ello, en la primera sección se describen y analizan las estadísticas judiciales provenientes de distintos órganos públicos. En la sección siguiente se da cuenta de los resultados cualitativos obtenidos a través de la investigación realizada, para finalizar con un conjunto de conclusiones. Especial mención se realizará a las medidas cautelares decretadas por ambas judicaturas, así como a las condiciones fijadas en las salidas alternativas aplicadas, la suspensión condicional de la dictación de la sentencia en sede familiar y la suspensión condicional del procedimiento en sede penal.

\section{LA VIOLENCIA INTRAFAMILIAR EN CIFRAS}

La primera parte de este estudio contempló el levantamiento de información estadística sobre casos de violencia doméstica desde 1999 hasta 2009, utilizando distintas fuentes de información. La idea era conocer la evolución y cambios que ha experimentado el tratamiento de la violencia intrafamiliar considerando las modificaciones legislativas de los últimos años.

${ }^{17}$ La muestra no es representativa, no obstante su selección fue al azar a través del sistema informático de causas del Poder Judicial en las demandas en tribunales de familia, mientras que los casos en sede penal fue realizada a través de una selección de casos terminados por suspensión y que se encontraban en los archivos de las respectivas fiscalías. 
La Tabla 1 muestra el número de causas de violencia intrafamiliar ingresadas y terminadas por tribunales de letras o civiles entre los años 1996 a 2005, con datos provenientes de dos fuentes distintas, el Servicio Nacional de la Mujer (SERNAM) y la Corporación Administrativa del Poder Judicial (en adelante CAPJ). Como se observa, hay, por una parte, algunas lagunas en el registro de la información y, por otra, discrepancias en las cifras de las fuentes consultadas, lo que se puede explicar por problemas en el registro de los datos.

TABLA 1

CAUSAS JUdiCIALES INGRESADAS Y TERMINADAS EN MATERIA DE VIOLENCIA INTRAFAMILIAR EN TRIBUNALES CIVILES Y DE LETRAS BAJO LA LEY N N $^{\circ} 19.325$

\begin{tabular}{|c|c|c|c|c|}
\hline \multirow[t]{2}{*}{ Año } & \multicolumn{2}{|c|}{ Causas ingresadas } & \multicolumn{2}{|c|}{ Causas terminadas } \\
\hline & Fuente 1 & Fuente 2 & Fuente 1 & Fuente 2 \\
\hline 1996 & 56.396 & & 40.312 & \\
\hline 1997 & 62.252 & & 53.198 & \\
\hline 1998* & & 10.827 & & 7.395 \\
\hline $1999 * *$ & & 57.350 & & 40.060 \\
\hline 2000 & & 71.349 & & 47.265 \\
\hline 2001 & 83.184 & 72.609 & 45.033 & 44.977 \\
\hline 2002 & 86.968 & 81.085 & 52.068 & 52.179 \\
\hline 2003 & 95.844 & 91.734 & 63.248 & 63.660 \\
\hline 2004 & 101.194 & 101.229 & 89.309 & 89.511 \\
\hline $2005 * * *$ & 71.598 & 79.951 & 41.471 & 75.830 \\
\hline
\end{tabular}

Fuente 1: SERNAM, Causas Judiciales Ingresadas en materia de violencia Intrafamiliar. En: <http://www. sernam.cl/cedocvi/basemujer/docs/CON(9)_20080711_104915.xls >. Visitado el 30 de octubre de 2009. Para los años 1996-1997 de datos procesados por el INE, Anuarios de Justicia y Compendio Estadístico.

Fuente 2: Elaboración propia con datos proporcionados por la Corporación Administrativa del Poder Judicial.

* Los datos están incompletos. No se registra información respecto de los tribunales de letras de las Cortes de Apelaciones de Arica, La Serena, Santiago.

** Es relevante hacer notar los problemas de registro. En algunas Cortes, como la de Valparaíso, tiene un número muy superior a términos que los registros de ingresos que indican para 1999 y el 1998 , ello se explica porque no se habían contabilizado los casos en esos períodos. En otra como la de Punta Arenas no se registra ningún caso terminado ese año en 1999, lo cual resulta extraño, y nuevamente pensamos que se explica por las deficiencias en el registro de información.

*** Se utilizó la información de INE, Anuario Estadístico 2006, Cuadro 43, Santiago, 2007, p. 201. 
Con todo, ambas fuentes relevan el aumento de la judicialización de las denuncias en tribunales desde la existencia de legislación específica (1994) y también se advierte que las divergencias de la información se van reduciendo a partir del año 2000.

En octubre de 2005 comienza la aplicación de la Ley $\mathrm{N}^{\circ} 19.968$ que crea los tribunales de familia, entregándoseles a estos juzgados el conocimiento de los casos de violencia intrafamiliar. La Tabla 2 muestra los ingresos a tribunales de familia y de letras con competencia en familia entre octubre de 2005 a junio de 2008 y se observa la misma tendencia al crecimiento de casos que se había observado bajo la Ley $\mathrm{N}^{\circ} 19.325$.

\section{TABLA 2}

FreCuENCIA DE INGRESOS DE CAUSAS POR VIOLENCIA INTRAFAMILIAR EN Tribunales de FAMILIA Y CON COMPETENCIA EN FAMILIA OCT. 2005 A 30 JUNIO DE 2008

\begin{tabular}{cccc}
\hline 2005 & 2006 & 2007 & 2008 \\
\hline 26.036 & 89.129 & 94.648 & 51.032 \\
\hline
\end{tabular}

Fuente: Elaboración propia con datos proporcionados por la Corporación Administrativa del Poder Judicial.

Ahora bien, nuestro foco no sólo fue medir cuántos casos ingresan y salen del sistema al año, sino que el objetivo fue conocer la forma en que éstos terminan, especialmente a partir del 2005, con la introducción de las salidas alternativas tanto en sede penal como familiar.

Los datos muestran, por ejemplo, que en el primer semestre de 2008 la principal forma de término es la sentencia definitiva con un $52 \%$, tal como se aprecia en la Tabla 3. Le siguen en segundo y tercer lugar, no dar curso a la demanda y la incompetencia con un $18,3 \%$ y $17,2 \%$, respectivamente.

TABLA 3

CAUSAS TERMinAdAS POR FORMAS DE TÉRMINO 2005, 2006, 2007 Y PRIMER SEMESTRE 2008

\begin{tabular}{|c|c|c|c|c|c|c|c|c|}
\hline \multirow{2}{*}{$\begin{array}{l}\text { Forma de } \\
\text { término }\end{array}$} & \multicolumn{2}{|c|}{2005} & \multicolumn{2}{|c|}{2006} & \multicolumn{2}{|c|}{2007} & \multicolumn{2}{|c|}{2008} \\
\hline & $\mathrm{N}$ & $\%$ & $\mathrm{~N}$ & $\%$ & $\mathrm{~N}$ & $\%$ & $\mathrm{~N}$ & $\%$ \\
\hline $\begin{array}{l}\text { Sentencia } \\
\text { definitiva }\end{array}$ & 3.587 & 22,5 & 17.054 & 38,8 & 30.527 & 48,9 & 35.445 & 52,0 \\
\hline $\begin{array}{l}\text { Sentencia de } \\
\text { término }\end{array}$ & 0 & & 0 & & 9 & 0,02 & 0 & \\
\hline
\end{tabular}




\begin{tabular}{|c|c|c|c|c|c|c|c|c|}
\hline \multirow{2}{*}{$\begin{array}{l}\text { Forma de } \\
\text { término }\end{array}$} & \multicolumn{2}{|c|}{2005} & \multicolumn{2}{|c|}{2006} & \multicolumn{2}{|c|}{2007} & \multicolumn{2}{|c|}{2008} \\
\hline & $\mathrm{N}$ & $\%$ & $\mathrm{~N}$ & $\%$ & $\mathrm{~N}$ & $\%$ & $\mathrm{~N}$ & $\%$ \\
\hline Avenimiento & 831 & 5,2 & 104 & 0,2 & 95 & 0,1 & 87 & 0,1 \\
\hline Transacción & 34 & 0,2 & 84 & 0,2 & 11 & 0,01 & 8 & 0,01 \\
\hline Conciliación & 1.004 & 6,3 & 239 & 0,5 & 114 & 0,1 & 66 & 0,09 \\
\hline Acumulación & 31 & 0,2 & 7 & 0,01 & 0 & & 3 & 0,004 \\
\hline Mediación & 1 & 0,006 & 2 & 0,004 & 0 & & 0 & \\
\hline No da curso & 1.496 & 9,3 & 7.717 & 17,5 & 10.989 & 17,6 & 12.522 & 18,3 \\
\hline Desistimiento & 2.229 & 13,9 & 4.466 & 10,1 & 4.696 & 7,5 & 3.526 & 5,1 \\
\hline Abandono & 573 & 3,5 & 2.379 & 5,4 & 2.202 & 3,5 & 1.388 & 2,0 \\
\hline Incompetencia & 1.034 & 6,4 & 6.670 & 15,1 & 8.465 & 13,5 & 11.779 & 17,2 \\
\hline Retiro & 741 & 4,6 & 1.037 & 2,3 & 21 & 0,03 & 178 & 0,2 \\
\hline Sin movimiento & 4.364 & 27,4 & 4.121 & 9,3 & 279 & 0,4 & 65 & 0,09 \\
\hline Otros motivos & 0 & & 0 & & 0 & & 3.056 & 4,4 \\
\hline Total & 15.925 & 100 & 43.886 & 100 & 62.397 & 100 & 68.123 & 100 \\
\hline
\end{tabular}

Fuente: Elaboración propia con datos proporcionados por la Corporación Administrativa del Poder Judicial.

Llama la atención el alto número de sentencias que se dictan en estos casos, pero nuestro convencimiento - a partir de conversaciones con jueces y abogados litigantes- es que dicha cifra incluye los casos terminados por suspensión condicional de la dictación de la sentencia, pues las estadísticas judiciales no incluyen esta categoría, lo que sería refrendado luego con los datos del Servicio de Registro Civil e Identificación. Este servicio cuenta con información detallada al respecto que muestra que esta forma de término supera casi cinco veces el número de condenas por actos de violencia, tal como se aprecia en Tabla 4.

\section{TABLA 4}

REGISTRO DE CONDENAS Y SUSPENSIONES DE LA DICTACIÓN DE LA SENTENCIA POR ACTOS DE VIOLENCIA INTRAFAMILIAR 2005-2009 EN REGISTRO CiVIL

\begin{tabular}{lrrrrrr}
\hline & 2005 & 2006 & 2007 & 2008 & 2009 & \multicolumn{1}{c}{ Total } \\
\hline Condenas & 889 & 1.057 & 1.114 & 1.314 & 578 & 4.922 \\
Multas & 419 & 1.642 & 1.672 & 2.262 & 2.292 & 8.287 \\
Medidas accesorias & & 2.449 & 2.879 & 3.935 & 2.100 & 11.363 \\
\hline
\end{tabular}




\begin{tabular}{lrrrrrr}
\hline & 2005 & 2006 & 2007 & 2008 & 2009 & Total \\
\hline $\begin{array}{l}\text { Suspensiones } \\
\text { Omisiones }\end{array}$ & 1.561 & 5.887 & 6810 & 7.013 & 4.444 & 25.715 \\
Total & & & & 50 & 291 & 341 \\
\hline
\end{tabular}

Fuente: Servicio de Registro Civil e Identificación.

De acuerdo a la información del Registro Civil el número de casos terminados sea por condenas, suspensiones o medidas accesorias es muy bajo en comparación con el número de términos de los tribunales de familia. Por ejemplo, en primer semestre del año 2008 terminaron 35.445 en sentencias de acuerdo a la información de la CAPJ y conforme a los datos del Registro Civil solo 2.292 terminaron en multas. Una posible explicación de la discordancia entre los términos y el número de condenas y suspensiones que lleva el Registro Civil podría mostrar la renuencia de los jueces de familia de remitir la información a dicho servicio para evitar que el ofensor "tenga sus papeles manchados"18.

Por otra parte, la categoría de incompetencia muestra el flujo de casos desde los tribunales de familia a las fiscalías, especialmente cuando los hechos se han calificado como maltrato habitual, categoría que dicho sea de paso no se contempla en las estadísticas del Registro Civil y que debe leerse a la luz de los datos que tiene el Ministerio Público. Estos datos, sin embargo, no dan cuenta de los casos que también "viajan" desde algunas fiscalías y a tribunales de familia -en lo que hemos denominado "este caso no es mí" 19 -, generándose discusión en torno a la oportunidad y forma en que el juez de familia debe apreciar la habitualidad, discusión en la cual incluso ha participado el Tribunal Constitucional ${ }^{20}$.

Por último, y a juzgar por la información revisada, aún quedan resabios del enfoque terapéutico de la Ley $\mathrm{N}^{\circ} 19.325$, pues todavía se registran casos que terminan por avenimiento, conciliación y transacción, vías no previstas en la legislación actualmente vigente.

Por su parte, los datos del Ministerio Público arrojan que la suspensión condicional del procedimiento representa la forma de término judicial más común, con un 26,4\% en el 2007 y un $31,9 \%$ en el 2008, según se advierte en la Tabla 5.

${ }^{18}$ El artículo 96 de la Ley No 19.968 de Tribunales de Familia establece que la resolución que apruebe la suspensión debe ser inscrita en el Registro Especial de Condenas por Actos de Violencia Intrafamiliar que para estos efectos llevará el Servicio de Registro Civil e Identificación.

19 Centro de Derechos Humanos, Informe Anual sobre Derechos Humanos en Chile 2009, Facultad de Derecho Universidad Diego Portales, Santiago, 2009, pp. 188-192.

${ }^{20}$ Ibíd., pp. 189-190. 
TABLA 5

Formas DE TÉRMINO CAUSAS VIOLENCIA INTRAFAMILIAR EN SEDE PENAL

\begin{tabular}{|c|c|c|c|c|}
\hline \multirow{2}{*}{ Tipo de término } & \multicolumn{2}{|c|}{2007} & \multicolumn{2}{|c|}{2008} \\
\hline & $\mathrm{N}$ & $\%$ & $\mathrm{~N}$ & $\%$ \\
\hline Sentencia condena & 6.877 & 7,3 & 10.329 & 9,0 \\
\hline Sentencia absolutoria & 174 & 0,1 & 346 & 0,3 \\
\hline Sobreseimiento definitivo & 1.119 & 1,2 & 2.176 & 1,8 \\
\hline Sobreseimiento temporal & 1.078 & 1,1 & 1.534 & 1,3 \\
\hline Suspensión condicional del procedimiento & 24.661 & 26,4 & 36.617 & 31,9 \\
\hline Acuerdo reparatorio & 348 & 0,3 & 271 & 0,2 \\
\hline Facultad para no investigar & 2,958 & 3,1 & 3.611 & 3,1 \\
\hline Subtotal términos judiciales & 37.215 & 39,9 & 54.884 & 47,8 \\
\hline Archivo provisional & 31.560 & 33,8 & 32.689 & 28,5 \\
\hline Decisión de no perseverar & 2.990 & 3,2 & 5.994 & 5,2 \\
\hline Principio de oportunidad & 13.922 & 14,9 & 13.604 & 11,2 \\
\hline Incompetencia & 1.899 & 2,0 & 1.259 & 1,0 \\
\hline Subtotal términos no judiciales & 50.371 & 54,0 & 53.546 & 46,7 \\
\hline Anulación administrativa & 94 & 0,1 & 57 & 0,04 \\
\hline Agrupación a otro caso & 5.189 & 5,5 & 5.857 & 5,1 \\
\hline Otras causales & 157 & 0,1 & 124 & 0,1 \\
\hline Otras causales de suspensión & 96 & 0,1 & 146 & 0,1 \\
\hline Subtotal otros términos & 5.536 & 5,9 & 6.184 & 5,3 \\
\hline Total & 93.122 & 100 & 114.614 & 100 \\
\hline
\end{tabular}

Fuente: Ministerio Público, Boletín Estadístico 2007 y 2008.

Llama la atención la alta proporción de casos que finalizan por salidas no judiciales, esto es, archivo provisional, principio de oportunidad, decisión no perseverar e incompetencia. Durante el año 2007 más de la mitad de los casos ingresados terminaron con este tipo de salidas (54\%), advirtiéndose una leve disminución en el año 2008 (46,7\%). Lo anterior contrasta fuertemente con la baja proporción de causas terminadas por condenas, un 7,3\% en el año 2007 y un $9 \%$ en el 2008.

\section{TRATAMIENTO Y RESPUESTA A LA VIOLENCIA INTRAFAMILIAR}

La segunda parte de este estudio consistió en la revisión y análisis de 140 carpetas de tribunales de familia y 148 de fiscalías de las Regiones V y Metropolitana con el fin de determinar, ahora desde una perspectiva cualitativa, el tratamiento y respuesta del sistema judicial a la violencia intrafamiliar. 
La revisión de las carpetas muestra que la mayoría de las víctimas son mujeres, tanto en sede penal $(88,5 \%)$ como en sede familiar (95\%), cuyas edades fluctúan entre 30 y 47 años. Si bien en ambas judicaturas se registran denuncias de hombres, de acuerdo a la información recabada, éstos suelen ser maltratados por otros hombres, ya sean hijos, hermanos, yernos o padres.

Sin perjuicio de ello, la nomenclatura utilizada por nuestro ordenamiento jurídico -actos de violencia intrafamiliar- muestra que los casos ingresan como problemas de maltrato al interior de la familia en un sentido genérico, lo que no releva la unidireccionalidad del fenómeno: hombres que maltratan a sus parejas o a otros miembros del entorno familiar. Concordamos en este sentido con Amorós cuando señala que las formas que utilizamos para denominar las cosas, los hechos, los fenómenos tienen altos componentes políticos ${ }^{21}$. En este caso dar un nombre genérico a aquello que es específico puede mantener en el imaginario colectivo la idea de "familia violentada", cuando en verdad se trata de violencia en contra de mujeres por parte de varones. Esto es especialmente evidente al momento de registrar y contabilizar casos de violencia intrafamiliar, pues no distinguen quiénes son los perpetradores y las víctimas.

Ahora bien, pese a que la violencia se dirige hacia la mujer, cuando existen otros miembros en la familia ellos también resultan afectados, especialmente si se trata de niños, niñas y adolescentes. De acuerdo a las carpetas revisadas, más de un $93 \%$ de las denunciantes en sede familiar tenía hijos/as, porcentaje que aparece más bajo en sede penal $(57 \%)^{22}$.

Por otra parte, la gran mayoría de las mujeres que denuncian la violencia lo hacen en contra de sus cónyuges, convivientes, ex cónyuges, ex convivientes o personas con las cuales se tiene o se tuvo una relación de pareja. Esta situación es similar en ambas judicaturas, aun cuando las proporciones son distintas, $49,3 \%$ vs. $34,4 \%$ cuando se trata de cónyuges y $21,4 \%$ vs. $27,7 \%$ cuando se trata de convivientes, en sede de familia y penal, respectivamente.

Los tipos de violencia que muestran los casos son múltiples: desde la violencia sicológica hasta la sexual pasando por las amenazas, la destrucción de enseres domésticos y la violencia física. En los tribunales de familia la violencia sicológica es la manifestación de más alto ingreso con un 43,7\%, la cual -de acuerdo a lo que consignan las demandas y partes policiales- consiste en insultos y humillaciones con expresión de afectación sicológica para las personas que denuncian.

Los relatos de los partes policiales y las denuncias trasuntan las construcciones genéricas que están a la base de los roles y las jerarquías familiares con expresiones tales como "no servís p'a la cama", "soi mala madre", "eres tonta, guatona, chancha", donde se manifiestan en forma explícita los roles estereotipados de género, tal como se aprecia de la siguiente información recabada de la revisión de carpetas.

${ }^{21}$ Amorós C., Conceptuar es Politizar. En: Género, violencia y derecho, Laurenzo, P., Maqueda, M.L. y Rubio, A. Coordinadoras, Tirant lo Blanch alternativa, Valencia, 2008, pp. 15-18.

${ }^{22}$ Esta situación puede explicarse porque las carpetas de los fiscales no consignan esa información. 
Según lo relatado en el parte-denuncia policial, la demandante es casada hace 15 años con el demandado, quien la agrede psicológicamente, lo que la menoscaba en su calidad de mujer y madre. Lo que motiva la denuncia, es que mantuvo una discusión con el demandado, quien le manifestó con palabras soeces que si no le cocinaba la iba agredir ${ }^{23}$.

Ella señala en su denuncia "Que es casada con xxx relación (de la cual) nacieron tres hijos los cuales se encuentran casados y que desde hace 3 años a la fecha, su cónyuge la insulta con palabras groseras menoscabándola en su calidad de madre y mujer, además le manifiesta a viva voz "que no me tienes la ropa planchada, no está listo el almuerzo y que se demora en prepararlo más de 5 horas", razón por la cual se encuentra afectada sicológicamente" 24 .

El segundo lugar lo ocupa la combinación entre maltrato verbal y psicológico más violencia física, la que alcanza al 16,4\%. En tercer orden de importancia están las denuncias por violencia verbal y sicológica más amenazas (de agredir a la denunciante, a los hijos/as o que el denunciado se suicidará), las que alcanzan a un 11,4\%.

Por su parte, en los casos investigados por el Ministerio Público, los partes policiales describen diversas manifestaciones de delitos (lesiones, amenazas, maltrato habitual, entre otros). También incluyen descripciones de categorías de conducta como "insultar en su condición de mujer y madre" o refiere lesiones sicológicas, maltrato infantil o violencia intrafamiliar a la mujer o adulto mayor, pero sin una narración fáctica que la acompañe, con lo cual no es posible establecer el tipo de hechos denunciados. Sin perjuicio de ello, la formalización por tipo de delito se concentra en lesiones menos graves en combinación con el delito de amenaza (55,6\%), seguidas de las amenazas (28,8\%). Cabe consignar que del total de carpetas revisadas el delito de maltrato habitual alcanza a menos de una décima parte $(8,3 \%)$.

\section{Medidas cautelares y de protección}

Otro foco del estudio fue indagar en relación a las medidas cautelares decretadas en ambas sedes y detectar sus diferencias y similitudes. Como sabemos, la finalidad de las medidas cautelares en la Ley $\mathrm{N}^{\circ} 19.968$ y en la 20.066 va más allá de la noción tradicional de asegurar el resultado de la acción y la eficacia de las resoluciones judiciales. Si bien algunas de las medidas contempladas en el artículo 92 de la Ley de Tribunales de Familia persiguen ese objetivo, la gran mayoría de ellas tiene otra finalidad: proteger a la víctima y a su grupo familiar, así como su subsistencia económica e integridad patrimonial, algo similar a lo que ocurre en materia penal.

En el caso de los tribunales de familia, la ley prevé un sistema que otorga una potestad cautelar amplia al juez, incluso sin la obligación de fundamentar la resolución que decreta las cautelares ${ }^{25}$. Se le entregan facultades para fijar las medidas que estime

\footnotetext{
${ }^{23}$ F- 37-2008 del Primer Tribunal de Familia de Santiago.

${ }^{24}$ F-1325-2007 del Tribunal de Familia de Viña del Mar.

${ }^{25}$ Artículo 22 de la Ley No 19.968 publicada en el Diario Oficial el 30 de agosto de 2004.
} 
pertinentes en tres situaciones específicas: en casos urgentes, cuando lo exija el interés superior del niño, niña o adolescente o cuando lo aconseje la inminencia del daño que se trata de evitar. Estas medidas podrán ser conservativas o innovativas, según si modifican el statu quo o no de la situación de hecho ${ }^{26}$.

La revisión de carpetas muestra que en la judicatura familiar las cautelares superan a las decretadas en sede penal $(49,2 \%$ vs. $28 \%)$. Las más frecuentes son la prohibición de acercarse a la víctima que alcanza a un $15,7 \%$, le sigue la combinación de ésta con la salida del hogar común con un $17,8 \%$ y otras de variada índole (rondas periódicas, tratamientos, terapias, etc.) que en total llegan a un $15,7 \%$.

En materia penal, se debe distinguir entre medidas cautelares que exigen la participación directa de un juez de garantía con la posibilidad de ser discutidas por la defensa y las medidas de protección. Estas últimas pueden ser adoptadas por el Ministerio Público sin intervención judicial, pues no comprometen los derechos de los imputados ${ }^{27}$.

Las carpetas revisadas muestran que no más allá de un tercio de los casos presentaba medidas de protección, y no fue posible vislumbrar los criterios usados por las fiscalías para determinarlas. De éstas, en una quinta parte de los casos se adoptó la medida de protección de ronda policial + contacto telefónico prioritario, y casi en un $10 \%$ se trató de contacto prioritario o llamadas telefónicas periódicas.

Por su parte, las medidas cautelares solicitadas por las fiscalías y decretadas por los jueces de garantía no superan el $28 \%$ de los casos y consisten principalmente en la combinación de salida del hogar común más prohibición de acercamiento + firma del imputado que alcanza a un $10,1 \%$.

Llama la atención que en 4 de los 30 casos en que se decreta la cautelar de prohibición de acercamiento se deja expresa constancia que esta medida es "sin perjuicio del derecho a visitas". Si bien se comprende la necesidad de hacer esta salvedad -probablemente en atención a la existencia de hijo/as comunes entre víctima y victimario- ella se realiza en términos tan generales que la interpretación y aplicación de la medida queda entregada a las partes. Ello reviste especial complejidad, especialmente si el régimen comunicacional ha sido regulado por un tribunal de familia, pues el cumplimiento de una decisión judicial implicará - de algún modo- la infracción de la otra.

La prevalencia de la cautelar de salida del hogar que se observa en ambas judicaturas se puede explicar porque -como vimos- la mayoría de las denuncias se hacen en contra de cónyuges o convivientes. La cautelar de abandono del hogar común aparece como una medida racional frente a los actos de agresión cuando las partes viven juntas y se busca que éstos cesen.

${ }^{26}$ Por ejemplo, si el ofensor es el marido de la víctima, el juez podrá decretar una prohibición de celebrar actos o contratos sobre un inmueble familiar, lo que tendrá por objeto mantener, conservar, el estado de hecho existente, esto es, la integridad del patrimonio familiar; o bien, podría innovar decretando el pago de alimentos provisorios.

${ }^{27}$ Fiscalía Nacional del Ministerio Público. Oficio 143. Instructivo general $\mathrm{N}^{\mathrm{o}} 11$ sobre atención y protección a las víctimas en el nuevo Código Procesal Penal. 12 de octubre de 2000. 
Cabe señalar, sin embargo, que esta medida es cuestionada por abogados litigantes quienes ven en ella un "proxy" del divorcio de los pobres ${ }^{28}$. No obstante, en este tipo de reflexión se omite considerar que las crisis de separación entre cónyuges o convivientes agudizan situaciones de conflicto e incluso desatan agresiones cuando uno de ellos no desea la separación. El Informe PNUD 2010 muestra que los hombres en proceso de separación experimentan malestar y desasosiego, por lo que el miedo a quedarse solos no les permite tomar la decisión o aceptarla cuando es un hecho ${ }^{29}$. Mientras menor es la disposición al cambio más aumenta el malestar, lo mismo que el miedo a enfrentar el aislamiento, lo cual "actúa como "una olla psíquica que explota ya sea en agresiones contra sí mismo, que se traducen principalmente en el abuso de sustancias o dependencias del alcohol, o en agresiones hacia otros hombres..."30. En ese contexto es factible que los hombres se sientan más inseguros y muestren respuestas más agresivas especialmente cuando no aceptan las nuevas condiciones en sus relaciones de pareja y carecen de capacidad de diálogo.

Como se puede apreciar, no hay grandes diferencias en el tipo de medidas cautelares decretadas en ambas judicaturas, la prohibición de acercamiento a la víctima y la salida del hogar común del ofensor son las más abundantes. Se observa, sin embargo, una importante diferencia en relación a la proporción de las medidas decretadas en una y otra sede $-49 \%$ en sede de familia versus $28 \%$ en sede penal-, diferencia que podría verse morigerada si a las cautelares penales se suman las medidas de protección dictadas por los fiscales que alcanzan a un $30 \%$.

\section{Condiciones de las salidas alternativas}

Por último, interesaba indagar acerca de las condiciones impuestas tanto a través de la suspensión condicional de la dictación de la sentencia en sede familiar como de la suspensión del procedimiento en sede penal. Cabe señalar que en ambos casos la ley prevé que las medidas cautelares puedan mutar a condiciones de estas salidas alternativas y se mantengan hasta un plazo no superior a un año.

En materia de familia pudimos constatar que una de las condiciones más comunes es el sometimiento a una terapia o tratamiento que alcanza casi a un tercio de los casos. La imposición de un tratamiento contra el alcoholismo corresponde a un 28,3\%, mientras que contra las drogas alcanza a un $6,4 \%{ }^{31}$. En algunos casos, a ellos se suman

${ }^{28}$ Krausz, A., ¿Violencia intrafamiliar: el nuevo divorcio de los pobres?, 10 de agosto de 2010. En: http:// blog.latercera.com/blog/akrausz/entry/violencia_intrafamiliar_el_nuevo_divorcio. [citado el 14 de septiembre de 2010]

${ }^{29}$ PNUD, Desarrollo Humano en Chile. Género: los desafíos de la igualdad. Santiago, 2010, pp. 95-97.

${ }^{30}$ Ibíd., pp. 100-101.

${ }^{31}$ Los datos citados por el PNUD muestran que en 2006, el 20\% de los hombres presentaba un abuso de consumo de alcohol en comparación con un $5 \%$ de las mujeres e indica que el alcoholismo es uno de los factores de riesgo de la violencia. PNUD, op. cit., p. 101. 
terapias sicológicas o psiquiátricas individuales para el control de impulsos $(8,4 \%)$ o terapias de pareja $(11,3 \%)$.

Por otra parte, la combinación de salida del hogar común más la prohibición de acercamiento llega a menos de una cuarta parte de los casos (22,8\%). La salida del hogar junto con otras medidas -como terapia o no repetir conductas violentas-alcanzan a un $28,2 \%$ de los casos, porcentaje ostensiblemente menor que al detectado en sede penal $(58,1 \%)$.

A nuestro juicio, en un $12 \%$ de casos terminados por suspensión condicional de la dictación de la sentencia esta salida no era procedente -o al menos su procedencia era altamente discutible - ya que los denunciados tenían denuncias anteriores por violencia en contra de la misma denunciante.

Ahora bien, en 20 de los 140 casos terminados por suspensión condicional de la dictación de la sentencia se abordan lo que hemos denominado "temas conexos", esto es, aquellos que surgen luego del quiebre familiar, especialmente cuando hay hijos/as. Se tiende a establecer, por ejemplo, el monto y formas de pago de la pensión de alimentos, así como la frecuencia e intensidad del régimen comunicacional. La combinación que se da con mayor frecuencia es la de alimentos + relación directa y regular, lo que se constata en 7 de los 20 casos.

Por su parte, en sede penal la salida del hogar en conjunto con la prohibición de acercamiento a la víctima es la combinación más frecuente $(58,1 \%)$, seguida por la prohibición de acercamiento más fijación de domicilio (47\%) y casi un $10 \%$ de las condiciones corresponde a lo que hemos denominado "pactos de no agresión”, como, por ejemplo, el compromiso del ofensor de no reiterar las conductas violentas. Cabe señalar, por último, que la información recabada no arroja datos sobre los llamados temas conexos.

Como se observa, tanto en sede penal como familiar el contenido de las salidas alternativas es bastante homogéneo. Las condiciones son prácticamente las mismas, solo se invierten las proporciones en que éstas aparecen. Mientras en familia priman los tratamientos y las terapias, en penal hacen lo suyo la prohibición de acercamiento y la salida del hogar común. Por otra parte, se observa que en muchos casos las condiciones establecidas en la suspensión condicional (ya sea de la dictación de la sentencia o del procedimiento) son las mismas medidas cautelares antes decretadas.

La revisión de los casos nos muestra que en general las personas que experimentan violencia acuden al sistema judicial pidiendo preferentemente que ésta cese. Todo indica que sus intereses no se encaminan necesariamente hacia la aplicación de una sanción al ofensor, pues ello no da a la víctima la seguridad que no se reiterará la misma conducta, bastando por tanto la aplicación de medidas que exijan el alejamiento del ofensor.

Esto lleva a preguntarse por el impacto real del sistema penal, en un contexto además de un alto porcentaje de salidas facultativas (46,7\% de acuerdo a los datos de 2008 del Ministerio Público). ¿Cuál es entonces el valor agregado de la intervención penal? Quizás sea su valor simbólico, pues se obtiene una respuesta del área más dura del derecho con una clara señal de que el sistema se hace cargo que se ha cometido un delito. Pero nos preguntamos hasta qué punto se hace cargo de este complejo fenómeno si la gran mayoría de los casos finaliza a través de salidas facultativas o alternativas. 


\section{Conclusiones}

El presente trabajo tuvo como objetivo indagar acerca del tratamiento y respuesta que reciben los casos de violencia intrafamiliar en el sistema de administración de justicia actual, específicamente en relación con las formas de término que ofrece la justicia de familia y la justicia penal.

Una de las primeras reflexiones que surgen a partir de este estudio se refiere a la calidad de la información con la que cuentan los organismos públicos, pues se advierten importantes deficiencias a nivel de registro y categorización de la información. Este tipo de problemas se observa particularmente en sede familiar donde no pudimos cuantificar el impacto de la suspensión condicional de la dictación de la sentencia con datos provenientes de la CAPJ, pues esta salida alternativa no es considerada como una categoría autónoma. Los datos recibidos y procesados hasta del primer semestre de 2008 no entregan información al respecto, y creemos a juzgar por la información del Servicio de Registro Civil que los casos terminados por esta vía se suman a las sentencias. Por otro lado, las estadísticas del Registro Civil no dan cuenta de la derivación de casos desde la justicia de familia a la penal cuando son considerados como maltrato habitual y procede la incompetencia, razón por la cual tampoco es posible saber con certeza el impacto de esta modificación introducida por la Ley $\mathrm{N}^{\circ} 20.066$.

Consideramos que la ausencia de datos fidedignos no permite diseñar y evaluar políticas públicas y judiciales desde la evidencia, sino por el contrario se hace desde intuiciones que conducirán inevitablemente a cometer errores que pueden costar muy caro a la legitimidad del sistema judicial y a los justiciables, más aún si ello se da frente a un fenómeno tan complejo como la violencia de género.

Ahora bien, y una vez hecha esta prevención, a la luz de los datos se puede constatar un aumento sostenido de la judicialización del fenómeno de la violencia intrafamiliar. Los primeros estudios del año 1996 dan cuenta en ingresos que promedian las 50.000 causas anuales, mientras que en el 2008 esta cifra prácticamente se duplica. Sin embargo, no hay que perder de vista que esta es una parte del fenómeno, lo que se ve, pues hay una cifra negra que desconocemos.

Desde el sistema de justicia penal se advierte que las principales formas de poner término a los casos son el archivo provisional como salida facultativa y la suspensión condicional del procedimiento como salida alternativa. Las sentencias condenatorias no alcanzan al $10 \%$ del total de las causas terminadas.

Creemos que los incentivos de gestión y carga de trabajo del sistema penal imponen soluciones rápidas, óptimas para el sistema, pero discutibles para los y las denunciantes, en que su participación en el proceso es escasa. La mecanización de las salidas alternativas con condiciones blandas y sin capacidad institucional de seguimiento soslaya los riesgos que las víctimas enfrentan. Encontrarnos además con condiciones de "pacto de no agresión” -aunque en baja proporción- era precisamente lo que se quería evitar con la dictación de la Ley $\mathrm{N}^{\mathrm{o}} 20.066$, y reitera la falta de sensibilización y entendimiento de las dinámicas de violencia. 
Podríamos decir que en el caso de la suspensión condicional del procedimiento se replican - guardando las debidas proporciones- los mismos argumentos que en sede familiar para preferir esta salida. De acuerdo a los resultados, se advierte que los casos de violencia intrafamiliar no llegan a juicio, eliminándose la necesidad con ello de llevar adelante una ardua investigación por parte del fiscal para probar, más allá de toda duda razonable, la culpabilidad del ofensor. Todo indica que para los operadores del sistema penal este tipo de asuntos son difíciles de abordar, con víctimas que en algunos casos prestan escasa colaboración y un alto grado de retractación ${ }^{32}$ (que en realidad no es la declaración que los hechos denunciados no hubieran ocurrido, sino que no se desea persistir en la persecución penal). Ello podría explicar la prevalencia de la suspensión condicional del procedimiento, por una parte, y de las salidas facultativas, por otra.

Este razonamiento es igualmente válido para los tribunales de familia con la salvedad de que estos muestran en sus respuestas un enfoque similar a lo que se resolvía bajo la Ley $\mathrm{N}^{\circ} 19.325$, esto es, la recomposición de los vínculos familiares y dar oportunidad al ofensor de cambiar su conducta a través de la rehabilitación. El desajuste sicológico y/o violento y la necesidad de reparar los lazos familiares está muy presente, y por ello se debe alertar sobre la existencia de redes que no sólo provean 'terapia familiar', sino también un trabajo de rehabilitación eficaz, reparación para las víctimas de la violencia que les permitan salir de un circuito violento, pues cuando median afecto y dependencia afectiva y/o económica las mujeres proporcionarán nuevas oportunidades a sus parejas para que "cambien". Si ese es el enfoque, entonces debe existir un monitoreo que lo acompañe; de otra manera, es dilatar la crónica de un fracaso anunciado.

En este sentido, no nos preocupa el bajo número de sentencias, especialmente cuando la ley prevé la posibilidad de una salida alternativa. Lo relevante es reconocer el tipo de trabajo y el tratamiento que se da a los casos. La importancia radica en tomarse el tiempo y adoptar las adecuadas condiciones, para que éstas sean concordantes con la gravedad del caso y la evaluación del riesgo del mismo. Hay, sin embargo, suficiente información anecdótica de víctimas que son presionadas a aceptar las suspensiones en sede familiar: se les advierte que los papeles del ofensor quedarán manchados, que irán a la cárcel o que no podrán pagar la pensión de alimentos.

Finalmente, si comparamos los efectos de la suspensión en sede familiar versus una suspensión en sede penal ahora en la persona del ofensor, todo indica que esta última le resulta menos gravosa. Esto se debe a que en materia penal no existe la anotación que aprueba la suspensión en ningún tipo de catastro, sin perjuicio del histórico del Ministerio Público, todo ello porque no hay reconocimiento de los hechos de violencia. La suspensión beneficia al imputado -y potencialmente a la denunciante, porque no se le sanciona con una multa- pues sus antecedentes no quedan 'manchados', cuestión que parece preocupar a los operadores. Paradójicamente, en los tribunales de familia si los hechos denunciados consisten en "zamarrear e insultar" y el proceso termina con una

32 Taladriz, M.J., San Martín, M.A. y Rodríguez, R., "La retractación en violencia intrafamiliar y su incidencia en el sistema procesal penal”, en: Revista Jurídica del Ministerio Público No 39, junio 2009, pp. 223-243. 
suspensión, el ofensor quedará en el registro histórico de actos de violencia intrafamiliar del Servicio de Registro Civil.

Todo indica que la intención de dar un trato más severo a la violencia doméstica conduce -en algunos casos- a resultados incongruentes. Por ello, y concordamos con Mera, sería un error buscar en el aumento del marco penal la respuesta más adecuada a la violencia, pues se queda con una reacción simbólica ${ }^{33}$, y como sostiene Larraín hay una excesiva confianza en la penalización y la judicialización dejando otros aspectos claramente de lado ${ }^{34}$.

Con la suspensión condicional del procedimiento, la intervención penal parece apuntar solo a detener la violencia y darle una oportunidad al ofensor para que no reincida, pero no se advierte seguimiento de las condiciones para asegurar su cumplimiento. Ello puede responder a la tosquedad del derecho penal como instrumento de control social, pero también una aplicación más bien mecánica de esta salida alternativa: se imponen siempre las mismas condiciones y sin ningún valor agregado.

Finalmente, es menester señalar que este estudio tiene limitaciones, por cuanto "el habla de la víctima” está mediado por su interpelación al sistema judicial -a través de escritos y registros que pueden producir información sesgada, y por ello, es importante mantener líneas de investigación que permitan hacer diagnósticos más acertados acerca de las necesidades de las víctimas. Las necesidades de las mujeres están influenciadas por el tratamiento burocrático que tienen los casos en cualquiera de las sedes, y ello se reproduce incluso en la instalación de tribunales especializados en violencia doméstica en el Estado de La Florida, EE.UU. ${ }^{35}$.

Consideramos que el tratamiento de la violencia doméstica exige una mirada integral y escapa a los cánones tradicionales del derecho penal el cual está concebido para mirar la foto, 'el último golpe', cuando se requiere que miremos la película completa ${ }^{36}$. Por lo mismo, ello tendrá distintas exigencias a la organización del aparato del Estado en su conjunto tanto judicial como de las instituciones colaboradoras y las que operan en él. Algo similar ocurrirá en los tribunales de familia.

Creemos que Chile avanza hacia mejores mecanismos de protección para enfrentar la violencia en contra de las mujeres, pero la eficacia de una ley y sus dispositivos descansan en una serie de factores y no la mera existencia de un cuerpo de normas. Por

33 Casas y Mera, op. cit.

${ }^{34}$ Larraín, Soledad, La situación de violencia de las mujeres en Chile. Legislación y Políticas públicas, consultoría nacional, Naciones Unidas. Disponible en: http://portal.unesco.org/geography/es/ files/11343/12436345861Resumen_ejecutivo_del_estudio_la_situacion_de_violencia_contra_las_mujeres_en_Chile._Legislacion_y_politicas_publicas.pdf/Resumen\%2Bejecutivo\%2Bdel\%2Bestudio\%2Bla\%2Bs ituacion $\% 2 \mathrm{Bde} \% 2 \mathrm{Bviolencia} \% 2 \mathrm{Bcontra} \% 2 \mathrm{Blas} \% 2 \mathrm{~B}$ mujeres $\% 2 \mathrm{Ben} \% 2 \mathrm{BChile.} \% 2 \mathrm{BLegislacion} \% 2 \mathrm{By} \% 2 \mathrm{Bp}$ oliticas\%2Bpublicas.pdf [citado el 23 de septiembre 2010]

${ }^{35}$ Coulter, M.L., Alexander, A. y Harrison, V., "Specialized Domestic Violence Courts: Improvement for Women Victims?” en Women E Criminal Justice, 16: 3, 2007, 91-106.

${ }^{36}$ Esta reflexión le pertenece a nuestro colega Matías Villalón con quien dialogamos durante el 2009 en las clases del Magíster de Derecho Penal de la Universidad Diego Portales sobre las limitaciones del modelo penal frente a la violencia doméstica. Santiago, diciembre de 2009. 
ello, vemos la necesidad de evaluar crítica y consistentemente los dispositivos creados y las políticas públicas que los acompañan. Solo a partir de medidas instadas desde la evidencia es posible avanzar por un buen camino.

\section{BIBLIOGRAFÍA}

Amorós, C., Conceptuar es Politizar. En: Género, violencia y derecho, Laurenzo, P., Maqueda, M.L. y Rubio, A. Coordinadoras, Tirant lo Blanch alternativa, Valencia, 2008, pp. 15-18.

Asún, R.; Fuenzalida, I.; Rodríguez, R. y Morales, M. Expectativas de las víctimas de delitos de violencia intrafamiliar más denunciados, Unidad Especializada en Responsabilidad Penal Adolescente y Violencia Intrafamiliar Fiscalía Nacional. Ministerio Público, Santiago, 2008.

Casas, L., "Ley N 20.066 sobre Violencia Intrafamiliar: ¿un cambio de paradigma?", en Anuario de Derechos Humanos 2006, Universidad de Chile.

Casas, L. y Mera, A., Violencia de Género y Reforma Procesal Penal chilena. Delitos sexuales y lesiones, Cuaderno de Análisis Jurídico, Serie de Publicaciones Especiales 16, Facultad de Derecho Universidad Diego Portales y el Centro de Estudios de Justicia para las Américas, Santiago, 2004.

Casas, L.; Dides, C. y Magaña, A., Estudio sobre la sistematización de información sobre el control de medidas y sanciones impuestas en virtud del artículo 5 de la Ley $\mathrm{N}^{\circ} 19.325$, Informe Final para el SERNAM, Santiago, 2001, mimeo.

Centro de Derechos Humanos, Informe Anual sobre Derechos Humanos 2009, Facultad de Derecho de la Universidad Diego Portales, Santiago, 2009.

Centro de Ética, Universidad Alberto Hurtado, Informe Ethos Violencia Intrafamiliar $\mathrm{N}^{\circ} 22$, 2000.

Coulter, M.L.; Alexander, A. y Harrison, V. "Specialized Domestic Violence Courts: Improvement for Women Victims?", en Women E Criminal Justice, 16: 3, 2007.

Fiscalía Nacional del Ministerio Público. Oficio 143. Instructivo general $\mathrm{N}^{\circ} 11$ sobre atención y protección a las víctimas en el nuevo Código Procesal Penal. 12 de octubre de 2000.

InStituto de LA Mujer, Estudio de seguimiento de la Ley de Violencia Intrafamiliar, Informe Final, Santiago, agosto de 1995, mimeo.

Krausz, A., ¿Violencia intrafamiliar: el nuevo divorcio de los pobres?, 10 de agosto de 2010. En: http://blog.latercera.com/blog/akrausz/entry/violencia_intrafamiliar_el_nuevo_divorcio. [citado el 14 de septiembre de 2010].

Larraín, S. La situación de violencia de las mujeres en Chile. Legislación y Políticas públicas, consultoría nacional, Naciones Unidas. Disponible en: http://portal.unesco.org/geography/ es/files/11343/12436345861Resumen_ejecutivo_del_estudio_la_situacion_de_violencia_contra_las_mujeres_en_Chile._Legislacion_y_politicas_publicas.pdf/Resumen\%2Beje cutivo $\% 2 \mathrm{Bdel} \% 2 \mathrm{Bestudio} \% 2 \mathrm{Bla} \% 2 \mathrm{Bsituacion} \% 2 \mathrm{Bde} \% 2 \mathrm{Bviolencia} \% 2 \mathrm{~B}$ contra$\% 2 \mathrm{Blas} \%$ 2Bmujeres\%2Ben $\% 2 \mathrm{BChile.} \% 2 \mathrm{BLegislacion} \% 2 \mathrm{By} \% 2 \mathrm{~B}$ politicas $\% 2 \mathrm{~B}$ publicas.pdf [citado el 14 de octubre 2010]

Ministerio Del INTERIOR, Encuesta Nacional de Victimización por Violencia Intrafamiliar y Delitos Sexuales 2008. En: http://www.seguridadpublica.gov.cl/files/presentacion_violencia_intrafamiliar_v2.pdf [citado el 23 de septiembre 2010].

Moltedo, C.; Silva, C.; Orellana, C. et al., Estudio sobre violencia doméstica en mujeres pobladoras chilenas, Santiago, 1989. 
OEA, Acceso a la Justicia para las mujeres víctimas de violencia en las Américas, Comisión Interamericana de Derechos Humanos, Washington, DC, 2007. OEA/Ser.L/V/II.Doc.68 20 enero de 2007.

PNUD. Desarrollo Humano en Chile. Género: los desafíos de la igualdad. Santiago, 2010.

Provoste, P., Violencia contra la mujer en la pareja: respuestas de la salud pública en Santiago de Chile, Serie Mujer y Desarrollo 85, CEPAL, Santiago, 2007.

República De Chile. Ley No 19.325 publicada en el Diario Oficial 4 de agosto de 1994.

LEY $\mathrm{N}^{\circ} 19.968$ publicada en el Diario Oficial 30 de agosto de 2004.

LEY No 20.066 publicada en el Diario Oficial 7 de octubre 2005.

Rioseco, L., Evaluación de la Ley de Violencia Intrafamiliar 19.325 y una propuesta para su modificación, Fundación Instituto de la Mujer, Santiago, 1997, mimeo.

Rodríguez, R., "Análisis estadístico descriptivo de los femicidios ocurridos en el año 2007”, Revista Jurídica del Ministerio Público $\mathrm{N}^{\circ} 35$, Julio 2008.

SERNAM, Femicidios 2008. En: <http://www.sernam.cl/portal/index.php/femicidios-2008> Visitado el 20 de noviembre de 2009.

Siegel, R., "Regulando la violencia marital" trad. Roberto Gargarella, en Derechos y Grupos desaventajados, Gargarella, R., comp., Yale Law School, Universidad de Palermo, y GEDISA Editorial, Barcelona, 1999.

TAladriz, M.J.; SAN MARTín, M.A. y RodríGUEZ, R. "La retractación en violencia intrafamiliar y su incidencia en el sistema procesal penal", en: Revista Jurídica del Ministerio Público $\mathrm{N}^{\circ} 39$, junio 2009. 